\title{
PÎVANEKA HELWESTA ZIMANÊ SERî (PHZS) JI BO DUZIMANAN: XEBATA TÊBARÎ Û PÊBAWERIYÊ
}

\section{Zafer AÇAR}

Bingöl Üniversitesi, Doğu Dilleri ve Edebiyatları Bölümü, Kürt Dili ve Edebiyatı ABD, Bingöl/Türkiye zafer13acar65@gmail.com, https://orcid.org/0000-0001-5323-9531

\section{Süleyman KASAP}

Van Yüzüncü Yıl Üniversitesi, İngilizce Eğitimi Bölümü, Van/Türkiye kasap_hakan@hotmail.com, https://orcid.org/0000-0001-8367-8789

\section{Article Types / Makale Türü \\ Research Article / Araştırma Makalesi}

\section{Received / Makale Geliş Tarihi}

04/02/2021

\section{Accepted / Kabul Tarihi \\ 24/04/2021}

DOI: https://doi.org/10.2679l/sarkiat.874798

Kurteya vê gotarê, di Kongreya ELCSSyê ya 03.02.202lan de hatiye pêşkeşkirin. 


\section{PÎVANEKA HELWESTA ZIMANÊ SERÎ (PHZS) JI BO DUZIMANAN: XEBATA TÊBARÎ Û PÊBAWERIYÊ}

\section{KURTE}

Armanca serekî ya vê xebatê pêşxistina pîvanekeke têbar û pêbawer e ku dikare helwesta kesên duziman raber zimanê wan yê serî diyar bike. Ev lêkolîna pîvanekê, serhev li ser 310 beşdarên duziman yên kurdî-tirkî yên li Tirkiyeyê -Bajarê Wanê- dijîn hatiye sepandin ku temenê wan di navbera16-65an de ye. Ji bo têbariya pîvanekê bê çespandin analîza fakterî ya keşfî û analîzeke pêbaweriyê hate çêkirin. Pîvanek li gorî encamên analîza fakterî ya keşfî̀ hate pêşxistin ku ew ji 31 maddeyên ji sê rehendan (hestyarî, xem û prestîja ziman, tercih û têrayiya ziman) pêk tê. Pîvana guncaniyê ya Kaiser-Mayer-Olkin (KMO) wekî .91 hate dîtin û bi testa Bartlett li hêza navbera têkiliya guherbaran hate nêrîn û di encamê de derhata kî-kare bi awayekî îstatîstîk $\left(\chi^{2}=3949,540\right.$, $\mathrm{p}=0.000)$ watedar hate dîtin. Bi vê encamê têderket ku analîza fakterî guncaw e. Barên fakterî yên bin rehendên faktera yekem di navbera 0.482 û $0.773 \mathrm{an}$ de, barên fakterî yên bin rehendên faktera duyem di navbera 0.335 û 0.720 an de û barên fakterî yên bin rehendên faktera sêyem jî di navbera 0,464 û 0,726an de nirxên xwe girtine. Tevî vê, qatjimara alfa ya bin rehendên PHZSyê bi rêzê, $0.872,0.885$ û 0.802 ne û qatjimara alfayê ji bo hemû maddeyên pîvanekê wekî 0.915 hatiye hesabkirin. Herwiha guncaniya îndeksê ya modelê û nirxê Chi-square (x2 $=924.69$, df $=428$, $\mathrm{p}=0.00000)$ watedar in. Nirxên îndeksa guncaniyê yên pîvanekê: $\mathrm{NNFI}=0.96, \mathrm{NFI}=0,93, \mathrm{CFI}=$ 0.96, RMSEA $=0.006$ hatine dîtin. Di encamê de derket ku pîvaneka ji bo vê xebatê hatiye pêşxistin têbar û pêbawer e û bi awayekî teorîk bingeheke wê heye ji bo nirxandina helwesta kesan raber zimanê wan yê serî.

Peyvên Sereke: Pîvanek, Zimanê serî, Duzimanî, Helwest

\section{MOTHER TONGUE ATTITUDE SCALE FOR BILINGUAL INDIVIDUALS: THE STUDY OF VALIDITY AND RELIABILITY}

\section{ABSTRACT}

The main aim of this study to develop a valid and reliable measurement scale that can be used to identify bilingual individuals' attitude towards their mother tongue. The scale research was applied to a total of 310 Kurdish-Turkish bilingual participants aged between 16-65 living in Van City -Turkey. An exploratory factor analysis and a reliability analysis were performed to determine the validity of the scale. The scale developed according to the results of explatory factor analysis consist of 31 items in total from three dimensions (Affective, Language anxiety and prestige, Language preference and proficiency). Kaiser-Mayer-Olkin (KMO) measure adequacy was found to be .91 and the strength of the relationship among variables by the Barlett test was found to be and Chi-square output was statistically significant $\left(\chi^{2}\right.$ $=3949,540, p=0.000)$. According to this results the factor analysis is fit. The scales' item factor of the sub-dimensions ranged from in the first dimension between 0.482 and 0.773 , in the second dimension between 0.335 and 0.720 , in the third dimension between 0,464 and 0,726. In addition, the total Cronbach Alpha reliability coefficient for the sub-dimensions of the scale in turn was $0.872,0.885$ and 0.802 and total Cronbach Alpha reliability coefficient of the scale was .915 . Moreover, the fit indices of model were exanimated and the Chi-square value $(\mathrm{x} 2=924.69, \mathrm{df}=428$, $\mathrm{p}=0.00000$ ) is significant. The fit index values of scale: $\mathrm{NNFI}=0.96, \mathrm{NFI}=0,93, \mathrm{CFI}=0.96, \mathrm{RM}-$ $\mathrm{SEA}=0.006$. It was concluded that the scale developed for this study was a valid, reliable and theoretically had a basis for evaluating the attitude of individuals towards their mother tongues.

Keywords: Scale, Mother Tongue, Bilingualism, Attitude 


\section{DESTPÊK}

Wekî qebûleke qiştî û terîfeke klasîk ziman amrazê pêwendiyê ye, lê di serdema modern de û îro di sehayên kontakta ziman û çandan yan jî carna pêvçûnên wan de û rewşên mîna rewşa kurdî de li Tirkiyeyê ku niha wekî zimanekî kêmepar digel zimanê zêdepar têkoşîna man û jiyanê dide; hingê êdî ziman ne tenê amrazeke pêwendiyê ye, em dikarin bejin ew niha nîşaneya terîf, nasnav û hêza civakan yan jî netewan e.

Xusûsen di dorwarên duziman de, bo nimûne li navendê bajarên mezin, ji ber perwerdeya giştî û berbelav û geşbûna teknolojiyê zimanên kêmepar yên ku bi awayekî fermî nehatine naskirin mîna zimanê kurdî li Tirkiyeyê, zimanê serî (zimanê dayîkê) yê qisevanê civaka kêmepar dikeve bin tesîra zimanê fermî (zimanê duyem) yan jî zimanê civaka zêdepar. Ji ber tesîr yan jî statuya zimanê fermî di zimanê yekem yê qisevanan de xirabûn çêdibe û hin hêmanên zimanê wî berew windabûnê diçin.

Carna zimanekî duyem dikare wisa raser be di civakekê de ku ew bike zimanê serî (dayikê) xira be, ku wekî windabûneke nepatolojîk ya parçe yan jî tevayiya zimanekî yê qisevanekî duziman tê pênasekirin. Di vê mijarê de xebatên ser xirabûna ziman ev bi qasî sê dehsal in bala komalgeha zanistî kişandiye ser xwe (Köpke û Schmid, 2004).

Îja di dorwarên duziman de digel bê statûbûna zimanê yekem jî, parastin û xwedîlêderketina wê ji aliye endamên wî zimanî ve nişana helwestekê dike ku ev diyardeyeke girîng e û hêjayî lêkolînê ye. Ji ber ku di tercîhkirina ziman de hokarên mîna ji ziman hezkirin, xema ziman, prestîja ziman, têrayiya zimanê qisevan, statûya ziman û yd. girîng in, dema helwesta qisevanan raber zimanê pêwendiyê hate vekolan ev hokar pirî caran dertêne pêş. Loma ev xebat armanc dike pîvanekek pêş bixe ku dikare helwesta qisevanan raber zimanê wan yê serî bipîve di dorwarên duziman de li dor wan hokarên navborî.

\section{HELWESTA ZIMAN Û HIN HOKARÊN SEREKÎ DI TERCÎHA ZIMAN DE}

Tiştekî pir asayî ye însan ji zimanê xwe hez bike û bi awayekî hestyarî xwe pê girêbide. Eger astengek yan jî tesîrên navîn û derîn nebin mirov piranî dixwazin zimanê xwe yê serî biparêzin, berdewam kin û biguhêzin ji bo nifşên bê. Îja hem ji bo zimanê xwe yê serî hem jî ji bo zimanên din mumkîn e însan xwedî hin fikir, bîr û baweriyan û helwestan bin. Bi taybetî jî di derbarê tercîhkirina ziman de li dorwarên duziman yan jî pirziman helwesta însanan raber zimanê pêwendiyê -çi zimanê wî/ê yê serî be çi jî zimanekî din be- ji ber hin hokaran şekil girtiye û kiriye ku ew bibe xwedî helwestekê. Dema em lîteraturê dinêrin di meseleyên têkilî ziman û nasnameyê de bo nimûne Fishman, yên têkilî plansaziya ziman de Spolsky û yên têkilî varyantên ziman de Giles amaje bi helwesta kesan kirine raber ziman ku ronî didine ser xebatên helwesta ziman. Wekî ku Garett (2010) derdibirre meseleyên helwesta ziman her cure diyardeyên sosyolînguîstîk û yên psîkolojiya civakî dihewîne yên têkilî mijarên wekî ka însan çawa xwe bi cih dikin bi awayekî sosyal û têkiliyên wan bi kes yan grûpên din re çawa nin.

Dema em li rewşa duzimanan dinêrin ku sehaya wan kesan e ku ew hem bi zimanê yekem hem jî bi zimanê duyem bi nisbeteke wekî hev yan jî nêzî hev dijîn, gelo ev kes xwedî xema ziman in, zimanê xwe yê serî yan jî yê duyem? Çawa ku Young (1991) derdibirre xema ziman derbarê zimanê biyanî de berbelav e di nav hînxwazên zimanê biyanî de ku em dikarin bêjin di dorwarên duziman de yê ku zimanê duyem raser e, berdewamkirina zimanê yekem jî dikare bibe sebeba xema ziman. Wekî xema ziman prestîja ziman jî kartêker e li ser bikaranîn û tercîhkirina zimanekî ku Hickey (2007) prestîja ziman zêdetir bi fermîbûn û statûya ziman ve girêdide. Wate, eger zimanek bi qanûnan bê parastin, bi awayekî fermî bê naskirin û di sehayên gel de jî bê bikaranîn hingê ew ziman xwedî prestîj e. Lê divê em vê jî bejin ku Trask (2007) bikaranîna zimanê fermî ji tercîhê zêdetir bi mecbûriyêtê ve ditêkilîne. Tevî van di tercîhkirina ziman de li dorhêlên duziman, bi taybetî jî di hingama axaftinê de, têrayiya ziman hokareke serekî ye ku însan pê zimanê xwe bi kar tînin. Di babeta motîvasyon û xwe-têriya kesan de Cummins (2000) dibêje; "kesekî di zimanê xwe yê pêşîn de jîr be ew di zimanekî din de jî jîr e dema ew wî zimanî hîn dibe". Herwiha li gorî Bandura (1982) xwe-têriya kesan asta motîvasyona wan diyar dike. Wate, kesên duziman bi qasî şiyana zimanê xwe yê serî dibin xwedî zimanekî din û dema xwe îfade dikin li gorî wê şiyanê tevdigerin. 
Îja ji ber giringiya hokarên me behs lê kirin ku kartêker in di çêbûna helwesta ziman de bi taybetî li dorwarên duziman, ev xebata ku wekî çêkirina pîvanekeke helwesta ziman e bêtir watedar dibe. Em hêvî dikin ew ê bibe alîkar ji lêkolînên vê biwarê re û ronî bide ser pirsiyarên têkilî mijarê.

\section{RÊBAZ}

Ev xebata wekî Pîvaneka Helwesta Zimanê Serî (PHZS) -ji bo Duzimanan- bi nav bûye, xebateke pêşxistina pîvanekekê ye. Armanca serekî ya vê xebatê ew e ku dixwaze pîvanekeke têbar û pêbawer pêş bixe ji bo tesbîtkirina helwesta kesan raber zimanê wan yê serî, di dorwareke duziman de.

\subsection{DÎZAYNA LÊKOLîNÊ}

Lêkolîn ji rêbazên lêkolînê yên kantîtatîv bi modela têgerînê dizayn bûye. Modela têgerînê ew lêkolîn e ku pê, taybetiyên beşdaran yên wekî; dên, dîtin, şiyan, helwest û ww. diyar dibin û li gorî lêkolînên din piranî nimûneyên ku xebat li ser çêdibe mezintir e. Armanca van lêkolînan bi giştî girtina fotografa rewşa heyî ya têkilî babeta lêkolînê ye û şayesandina wê ye (Fraenkel \& Wallen \& Hyun, 2012).

\subsection{GRÛPA LÊKOLÎNÊ}

Grûpa lêkolînê ya xebata me 310 kesên duziman bûn ku ew li Bajarê Wanê dijîn. Nimûneyên xebata me digel ku tirkî dizanin û ji dibistanê hînbûne, ew kurdîzar in (dikarin kurdî biaxivin û bixwînin) û zimanê wan yê serî (zimanê dayikê) kurdî ye. Ji bo ku nifûsa lêkolîna me kurdîzarên bajarê Wanê bûn ji bo ku em bigihêjine wan me wekî teknîka nimûnedanê; teknîka 'giloka berfê' bi kar anî. Teknîka 'Giloka Berfê' yan jî ya 'Zincîreyî' ya nimûnegirtinê, dema xwegihandina yekeyên ji nifûsê zehmet e yan jî dema agahiyên derbarê nifûsê (mazinahî û kûrahiya agahiyê û ww.) kêm dimîne ev teknîk bi kar tê (Baltac1, 2018: 253 -ji Patton, 2005).

Li aliyekî din, ji 310 kesên nimûneya xebata me 220 kes mêr bûn (\% 70.96) û 90 kes jî jin bûn (\% 29.03) û li gorî temenê xwe nimûneyên me; di navbera 16-65an de wekî pênc kategorî bûn; ' $16-25$, 26- 35, 36-45, 46-55, 56-65'.

\subsubsection{AMADEKIRINA AMÛRA BERHEVKIRINA DANEYAN}

Pîvaneka helwesta ziman ya kesane, ji bo helwesta kesan ya têkilî tercîha ziman diyar bike hatiye pêşxistin ku zimanê wan kesan yê serî kurdî ye. Ji bo vê, piştî têgerîna nav lîteraturê hewzeke maddeyan hate amadekirin û ev li ser beşdarên duziman hate sepandin. Ev madddeyên di vê formê de, ji bo em bizanin ka çiqas guncaw e helwesta beşdarên duziman bipîve li ser tercîha zimanê wan yê pêwendiyê û ka zimanê maddeyên wê çiqas fêmbar e, ji sê pisporên sehayê hate pirsin. Piştî hişyarî û pêşniyarên pisporan li ser maddeyan destkarî çêbû û wekî 35 pirsan şiklê formê yê dawî hate dayîn. Forma pîvanekê ji kategoriyên; "Qet Ne Wisa ye" (1), "Ne Wisa ye" (2), "Ne Erê Ne Na" (3), "Wisa ye" (4), "Bi Temamî Wisa ye" (5) pêk tê. Li gorî vê, pûanê berz nîşan dide ku helwesta ziman ya takekes berz e.

\subsubsection{PROSESA AMADEKIRINA DANEYAN JI BO ANALÎZÊ}

Daneyên ku li dor lêkolînê bi amûra komkirina daneyan hatine komkirin, li ser îstiqameta hin faraziyen diyarkirî li gor guncaniya wan ya ji bo analîza fakterî hatine vekolan. Ev; mezinahiya nimûneyê, daneyên winda/jidestçûyî, normalî, rasteyî, nirxên serik û fakterîbûna Ryê ne. Pêşî divê mezinahiya nimûneyê bê vekolan ka ji bo analîza fakterî guncaw e yan na. Li ser mezinahiya nimûneyê ji bo analîza fakterî di navbera lêkolîneran de rêkeftineke tam tuneye (İlhan û Çetin, 2014). Lê di lîteraturê de tê parastin ku divê jimara beşdaran 3 heta 6 carî jimara maddeyên pîvanekê be, 200 beşdar ji bo analîza fakterî guncaw in û 
500 beşdar jî heta bêjî baş in (Cattell, 1978). Bi zêdebûna beşdaran binyata fakterî bêtir diyar dibe lê ev rewş dema jimara beşdaran gihişte 5 qatî jimara sercem ya maddeyan dikare bê qebûlkirin (Stevens, 2002; Gorsuch, 1983). Dema seta daneyan ya vê xebatê ya li ser 310 kesan hatiye sepandin hate vekolan, tu daneyeke jidestçûyî nehate tesbîtkirin. Bi mebesta testkirina normaliya seta daneyan û rasteyîya wê pûanên sercem hatine kontrolkirin ka ew normal belav bûne yan na. Qatjimarên asîmetrîbûnê û pehnbûnê hatine nirxandin, testên normaliye hatine kirin. Bi mebesta tesbîtkirina nirxên nelê/ters ka di seta daneyan de hene yan tunene, pûanên z yên her yek guherbarekê hate vekolan. Hate dîtin ku pûanên z yên aîdî guherbaran di nav neqeba \pm 3.00 an de ye. Paşê bi mebesta kontrolkirina hemû guherbaran ka ew di guherbarên geleyî de nirxên serik in yan na, dûrbûna Mahalonobis hate hesabkirin û nirxên her guherbarî hate vekolan. Hate dîtin ku di seta daneyan de nirxên nelê/ters tunene. Bi mebesta pêkanîna kontrola Ryê ka dikare fakterî be nirxê KMO (Kaiser-Meyer-Olkin) û encama Testa Bartlett hate vekolan. Nirxê KMO .91 hate dîtin û encama Testa Barlett ya ku normaliya pir guherbariyê test dike jî $\left(\chi^{2}=3949,540, p=0.000\right)$ watedar derketiye. Dema em bala xwe bidine vê encamê em dikarin bejin ku dane guncaw in ji bo çêkirina analîza fakterî.

\subsubsection{ANALIIZA DANEYAN}

PHZS li ser beşdaran hate sepandin û ji bo xusûsiyetên pîvandinan bê derxistin analîza îstatîstîkî hate kirin. Ji bo ku em têbariya binyadî ya PHZSyê vekolin û binyata fakterî ya pîvanekê bînine der me 'Analîza Fakterî ya Keşfî' (explatory factor analysis) çêkir. Bona vê, mezinahiya nimûneyê ji bo AFKyê guncaw hate dîtin. PHZS ji 35 maddeyan pêk hatiye û ew li 310 beşdaran hatiye sepandin.

Dema AFK hate sepandin ji bo ku em diyar bikin bê ka kîjan teknîka zivirandinê ew ê bê sepandin, me teknîka zivirandinê ya 'dircet oblimin' bi kar anî. Herwiha ji bo em nirxên korelasyonê vekolin jî me dîsa teknîka direct oblimin sepand. Piştî vê zivirandinê hate dîtin ku di navbera bin rehendên pîvanekê de têkiliyeke nizm heye. Ji vê keşfê hate fêmkirin ku bin rehendên pîvanekê ji hev serbixwe ne. Bi qasî ku tê pêşniyazkirin di navbera bin rehendên kêm-têkilî de yan jî yên ji hev serbixwe de divê 'varimax' bê bikaranîn (Tabachnick û Fidell, 2007). Ji ber vê dema analîza fakterî ya keşfî hate kirin, wekî rêbaza fakterbûnê analîza pêkhênên bingeh, wekî rêbaza zivirandina fakterî jî ji rêbaza çik zivirandinê varimax hate sepandin. Ji bo pêbaweriya pîvanekê bê pêkanîn jî, ji tevahiya pîvanekê re bona her fekterekî qatjimara nav-hevgiriyê ya Cronbach Alfa û korelasyona maddeyê ya sercem hate hesibandin.

Ligel vê, bi alîkariya amûra komkirina daneyan, daneyên ku bi nivîskî hatine girtin; analîza wan bi programa paket SPSS 25 û programa LISSRLL 8.7 hatiye kirin.

\subsubsection{TESBÎT}

Di vê beşê de agahiyên derbarê pêbawerî û têbariya Pîvaneka Helwesta Ziman ya Kesane ew ê bên dan.

\subsubsection{TÊBARIYA BINYATÎ}

Ji bo têbariya binyatî ya PHZSyê bê diyarkirin analîza fakterî ya keşfî̀ (AFK) û analîza fakterî ya rasteder (AFR) hate kirin.

\subsection{ANALÎZA FAKTERÎ YA KEŞFî (AFK)}

Ji bo em têbariya binyatî ya PHZSyê tesbît bikin û barên fakterî yên maddeyan diyar bikin analîza fakterî ya keşfî̀ hate kirin. Ji bo guncawiya analîza fakterî ya keşfî bê diyarkirin qatjimara Kaiser-Meyer-Olkin (KMO) û testa Barlett Sphericity hate sepandin. Di encamê de nirxê KMO .91 hate dîtin û encama testa Bartlett ya ku normalbûna pir-guherbariyê test dike jî wekî $\left(\chi^{2}=3949,540, p=0.000\right)$ watedar hate dîtin. Bi vê encamê têderket ku analîza fakterî guncaw e. 
Di encama analîza fakterî de hate dîtin ku ew maddeyên ku xwe-nirxê wan ji 1ê mezintir in di 6 fakteran de kom bûne. Ev maddeyên ku di 6 fakteran de kom bûne, hate dîtin ku bi qasî \%56,521 yê pîvanekê îzah/ xuya kirine. Di analîza fakterî ya keşfî̀ de wisa hatiye derbirrîn ku divê barên fakterî yê maddeyan li ser 0,30yî be (Floyd û Widaman, 1995; Tabachnick û Fidell, 2007). Di analîza fakterî de ew maddeyên ku nirxê barê wan li bin 0.30yî ne û maddeyên ku bar nebûne ji analîzê hatine derxistin. Di encama analîza fakterî de biryar hate dayîn ku madde li ser sê fakteran bêne komkirin. Di Tabloya 1em de taybetiyên maddeyan yên têkilî encanmên AFKyê yên dubarekirî hatine pêşkeşkirin. Di encama analîza AFKyê de xwe-nirxên bi dest ketine û sedeyên varyansan yên sercem yên ku xuya bûne di Tablo 1ê de hatine dayîn û encama xêzegirafîka scree di Şeklê 1ê de hatiye nîşandan.

Tablo 1: Analîza Fakterî ya Keşfî̀ û Encamên Xwe-nirxên Derhatî

\begin{tabular}{|l|l|l|}
\hline & Encamên xwe-nirx yên AFKyê & Varyansa sercem ya derhatî \\
\hline Rehendê Iem & 9.448 & 17,370 \\
\hline Rehendê IIem & 2,879 & 15.920 \\
\hline Rehendê IIIyem & 1,800 & 12.279 \\
\hline
\end{tabular}

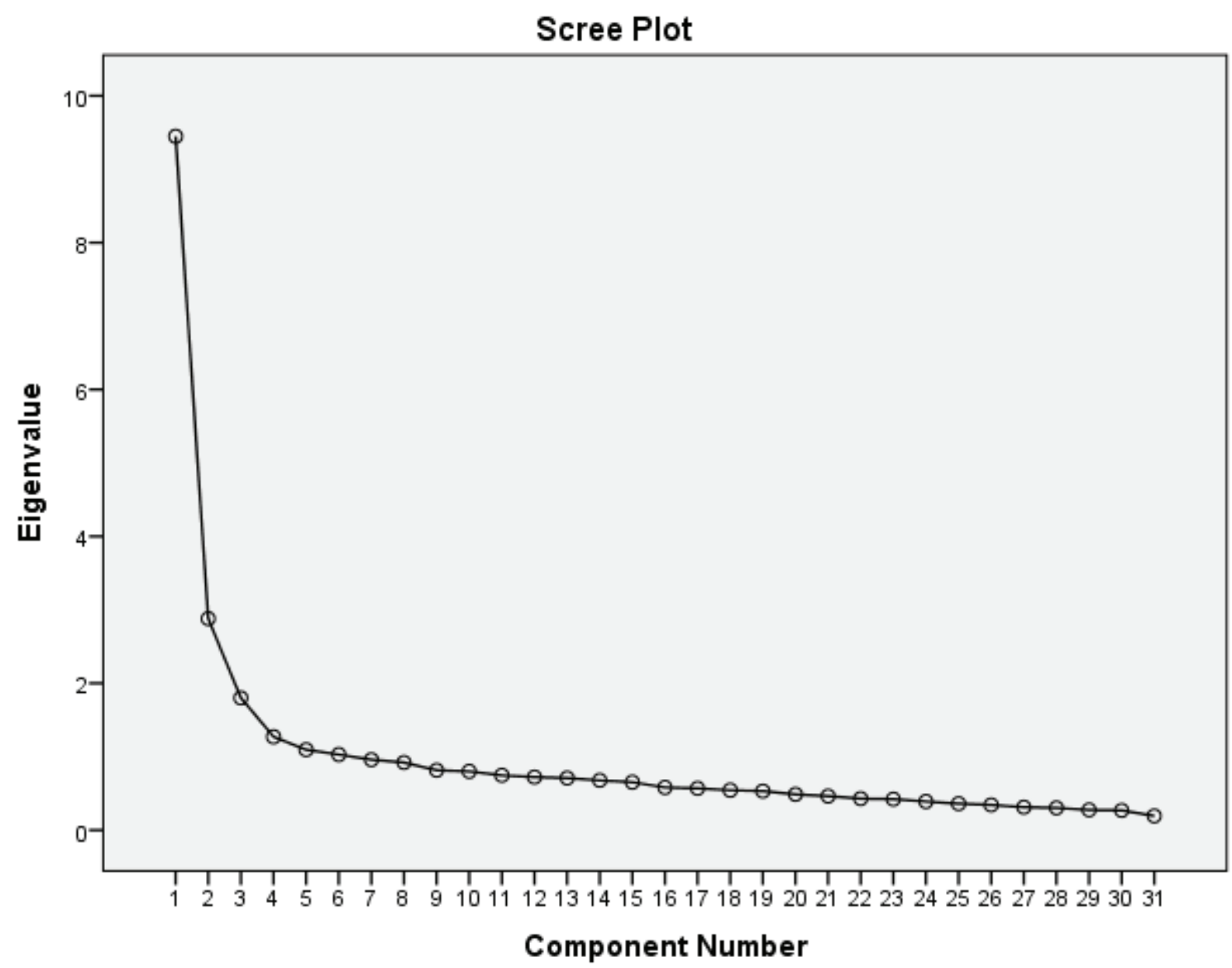

Şeklê 1em: Xêzegirafîk

Dema em berê xwe didine Tabloya 1em, em dibinin ku di encama analîza fakterî ya keşfî de madde li sê rehendan kom bûne. Dikare bê gotin ku; rehendê yekem \% 17,370yê varyansa sercem ya pîvanekê, rehendê duyem \% 15,920ê varyansa sercem ya pîvanekê û rehendê sêyem \% 12,279ê varyansa sercem ya pîvanekê îzah dike. Maddeyên ku di van hersê rehendan de kom bûne \%45.569ê varyansa sercem îzah dikin. Dema em bala xwe didine encama analîza faktorî, em wisa hizir dikin ku divê pîvanek bibe sê rehendî. Di Tabloya 2em de madde û barên fakterê wan hatiye dayîn. 
Tablo 2:

Fakterên di Encama AFKê de Çêbûne û Barên Fakteran

\begin{tabular}{|c|c|c|c|c|}
\hline Fakter 1: Hestyarî & Cronbach Alfa $=0.872$, & \multicolumn{3}{|c|}{ Varyansa derhatî $=\% 17,370$} \\
\hline & & \multicolumn{3}{|c|}{ Barê Fakterê } \\
\hline & & 1 & 2 & 3 \\
\hline \multicolumn{2}{|c|}{$\begin{array}{l}\text { 23. Li malê ez bi dê û bavê xwe re yan jî ehle malê re axaftina bi kurdî tercîh dikim ew } \\
\text { bi tirkî bikarin biaxivin jî. }\end{array}$} & .482 & & \\
\hline \multicolumn{2}{|c|}{ 25. Ez bi kurdî diaxivim, malbat û hevalên min kêfxweş dibin. } & .641 & & \\
\hline \multicolumn{2}{|c|}{$\begin{array}{l}\text { 26. Ez wisa hîs dikim ku ew ê bibe zirareke mezin eger ez nikaribim bi kurdî biaxivim, } \\
\text { bixwînim û binivîsim. }\end{array}$} & .632 & & \\
\hline \multicolumn{2}{|c|}{ 27. Mihefezekirina zimanê min ê dayikê ji bo min muhîm e. } & .733 & & \\
\hline \multicolumn{2}{|c|}{ 28. Ez bi kurdî baş diaxivîm berî ku ez diçûme mektebê. ${ }^{1}$} & .545 & & \\
\hline \multicolumn{2}{|c|}{ 29. Çendî xeta û kêmasiyên min hebin jî ez axaftina kurdî tercih dikim. } & .668 & & \\
\hline \multicolumn{2}{|c|}{$\begin{array}{l}\text { 32. Dema ez bi malbata xwe re me li ciyên fermî (wekî; mekteb, kargeh, civîn, banka, } \\
\text { pirtûkxane, mixaze, avahiyên îdarî) ez dixwazim bi wan re kurdî biaxivim. }\end{array}$} & .673 & & \\
\hline \multicolumn{2}{|c|}{ 33. Ez ji gelê kurd û çanda kurdan hez dikim. } & .723 & & \\
\hline \multicolumn{2}{|c|}{ 34. Ez bêtir rehet im dema ez bi kurdî diaxivim. } & .679 & & \\
\hline \multicolumn{2}{|l|}{ 35. Ez ji xwendina kurdî hez dikim. } & .659 & & \\
\hline & & \multicolumn{3}{|c|}{ Barê Fakterê } \\
\hline & & 1 & 2 & 3 \\
\hline Fakter 2: Xem û Prestîja Ziman & Cronbach Alfa $=0.885$ & \multicolumn{3}{|c|}{ Varyansa derhatî $=\% 15.920$} \\
\hline \multicolumn{2}{|c|}{ 1. Dema ez bi kurdî diaxivim (xeber didim) endîşe dikim ku ez ê xeta bikim. } & & .696 & \\
\hline \multicolumn{2}{|c|}{ 2. Dema ez bi kurdî diaxivim ditirsim ew ê bi min bikenin. } & & .720 & \\
\hline \multicolumn{2}{|c|}{ 3. Ew ên zimanê wan yê serî wekî yê min e, ez bi wan re bi zehmetî pêwendiyê datînim. } & & .592 & \\
\hline \multicolumn{2}{|c|}{$\begin{array}{l}\text { 5. Ez bi kurdî naxivim çunkî eger ez biaxivim însanên din ew ê bi nebaşî berê xwe bidine } \\
\text { min. }\end{array}$} & & .691 & \\
\hline \multicolumn{2}{|c|}{ 6. Ez bawer im ku axaftina bi kurdî ji bo min ew ê ne baş be. } & & .565 & \\
\hline \multicolumn{2}{|c|}{ 7. Li gorî min divê meriv pêşî zimanê fermî yê welatê lê dijî hîn be. } & & .516 & \\
\hline \multicolumn{2}{|c|}{ 8. Ku kurdî hatibe pirsîn jî ez carna bersivên tirkî didim. } & & .573 & \\
\hline \multicolumn{2}{|c|}{ 13. Ez tenê eger mecbûr bimînim bi kurdî diaxivim. } & & .604 & \\
\hline \multicolumn{2}{|c|}{$\begin{array}{l}\text { 14. Ez ditirsim dema ez kurdî biaxivim hevalên min ên kurdiya wan baş in, ew ê bi min } \\
\text { bikenin. }\end{array}$} & & .613 & \\
\hline \multicolumn{2}{|c|}{ 15. Ez ne bawer im axaftina bi kurdî ew ê statuyeke baştir bide min li Tirkiyeyê. } & & .335 & \\
\hline \multicolumn{2}{|c|}{ 16. Ez bi tirkî diaxivim, malbat û hevalên min kêfxweş dibin. } & & .445 & \\
\hline \multicolumn{2}{|c|}{$\begin{array}{l}\text { 17. Eger ez bi zimanê xwe yê serî neaxivim, nexwînim û nenivîsim, ew ê nebe kêmasi- } \\
\text { yeke mezin di jiyana min de. }\end{array}$} & & .480 & \\
\hline \multicolumn{2}{|c|}{ 18. Eger ez tirkî baş bizanibim, ev bes e ji bo jiyana min ya li Tirkiyeyê. } & & .393 & \\
\hline \multirow{3}{*}{\multicolumn{2}{|c|}{$\begin{array}{l}\text { 19. Dema ez bi malbata xwe re me li ciyên fermî (wekî; mekteb, kargeh, civîn, banka, } \\
\text { pirtûkxane, mixaze, avahiyên îdarî) ez dixwazim bi wan re tirkî biaxivim. }\end{array}$}} & & .543 & \\
\hline & & \multicolumn{3}{|c|}{ Barê Fakterê } \\
\hline & & 1 & 2 & 3 \\
\hline Fakter 3: Tercîh û Têrayiya Ziman & Cronbach Alfa $=0.802$ & Vary & erhatî & 279 \\
\hline 11. Ez bi tirkî difikirim paşê bi kurd & jim. & & & .577 \\
\hline 20. Ez hestên xwe bi tirkî baştir îfad & & & & .631 \\
\hline
\end{tabular}

1 - Çendî ev madde wekî ne hestiyar bixuyê jî piştî nirxandinên digel pisporên mijarê em gihîştine wê qeneetê ku; ji bo ku bîrbirineke dema berî û poşmaniyeke dema niha dihewîne ev madde dikeve bin kategoriya îfadeyeke hestiyar. 


\begin{tabular}{|l|l|l|}
\hline 21. Kurdiya min nebaş be jî ez rehet im dema li pêş însanan kurdî diaxivim. & .464 \\
\hline 22. Li mektebê bi hevalên xwe yên kurd re ez axaftina kurdî tercîh dikim & & .550 \\
\hline 24. Ez pêşî bi kurdî difikirim paşê bi tirkî dibêjim. & & .550 \\
\hline 30. Ez hêstên xwe bi kurdî baştir îfade dikim. & & .726 \\
\hline 31. Di her firsendê de, kengê dibe bila bibe ez zimanê xwe yê serî diaxivim. & .606 \\
\hline \multicolumn{2}{|c|}{ VARYANSA SERCEM YA DERHATî } & $\mathbf{\%} \mathbf{4 5 , 5 6 9}$ \\
\hline
\end{tabular}

Wekî ji Tabloya 2em diyar dibe, dema li barên fakterî yên maddeyan, binyatên teorik û naverokî yên maddeyan yên ji encama analîza fakterî bi dest ketine bê nerîn, faktera yekemîn wekî Hestyarî (H), faktera duyemîn wekî Xem û Prestîja Ziman (XPZ) û faktera sêyemîn jî wekî Tercîh û Têrayiya Ziman (TTZ) hate binavkirin. Fakterê yekem di bin rehenda xwe de \%17,370 ji sercema varyansê îzah dike û ji 10 maddeyan pêk tê. Wekî ku tê dîtin; barên fakterî yên bin rehendên faktera yekem di navbera 0.482 û 0.773 an de nirxê xwe girtine. Faktera duyem di bin rehenda xwe de \%15.920 yê sercema varyansê îzah dike û ji 14 maddeyan pêk tê. Barên fakterî yên bin rehendên faktera duyem di navbera 0.335 û 0.720 an de nirxên xwe girtine. Faktera sêyem jî di bin rehenda xwe de \%12.279ê sercema varyansê îzah dike û ew ji 7 maddeyan pêk tê. Barên fakterî yên bin rehendên faktera sêyem jî di navbera 0,464 û 0,726 an de nirxên xwe girtine. Divê em bejin ku nirxên barê fakterî yên 0.35 û li ser ketine ber nirxandinê.

Di analîza fakterî ya keşfî̀ de, tê dîtin ku maddeyên pîvanekê \%45,569ê sercema varyansê îzah dike. Dema em li qatjimarên korelasyonan dinêrin yên navbera bin rehendên ji pîvanekê bi dest ketine, em dibînin ku têkiliyeke nizm jê derketiye. Qatjimarên korelasyonê yên navbera bin rehendan; korelasyona di navbera bin rehenda yekem û bin rehenda duyem wekî 0.337 , korelasyona di navbera bin rehenda yekem û bin rehenda sêyem wekî - 0.231 û korelasyona di navbera bin rehenda duyem û bin rehenda sêyem jî -0.290 hatiye hesabkirin. Dema em bala xwe didine encamên van korelasyonan, têdertê ku di navbera bin rehendên pîvanekê de têkiliyeke nizm heye. Ji ber vê, ji rêbazên çik zivirandinê rêbaza varimax hate bikaranîn.

\subsection{ANALîZA FAKTERÎ YA RASTEDER}

Bona em rastiya binyata ku ji sê bin rehendan û 31 maddeyan pêk tê ya ku ji encama analîza fakterî ya keşfî̀ derketiye test bikin, me analîza fakterî ya rasteder sepand (AFR). Nirxên îndeksa lêhatinê ya têkilî PHZSyê di Tabloya 4em da hatiye dayîn.

Dema ev binyat hate testkirin ki-kare, dereceya 'ki-kare/serbestî̀'yê û îndeksên xweş-lêhatinê hatine hesabkirin ku ew di Tabloya 4em da hatine pêşkeşkirin. Herwiha di Tabloyê de ji bo îndeksan, pîvekên nirxandinê yên li gorî Schermelleh-Engel, Moosbrugger û Müller (2003) hatine qebûlkirin cih girtine.

Tablo 4: Encamên AFRyê yên Aîdê Binyata Sergirtî ya Sê Rehend

\begin{tabular}{|l|l|l|l|l|l|l|}
\hline Model & $\dot{\div}^{2}$ & $\dot{\mathbf{2}}^{\mathbf{2}} \mathbf{d s}$ & NNFI & NFI & CFI & RMSEA \\
\hline Binyata Sê Fakterî & 924.69 & 2,16 & 0,96 & 0,93 & 0,96 & 0,06 \\
\hline Pîvek & 3,0 & $\geq 0,95$ & $\geq 0,95$ & $\geq 0,95$ & $\leq 0,08$ & \\
\hline
\end{tabular}

Nirxên t yên ku ji encama AFRyê bi dest ketine ji pîvaneka sê rehend ya ji encama AFKyê derketiye di Tabloya 5em de cih girtine. Nirxê t yê ku ji encama AFRyê bi dest ketiye di bin rehenda yekemin de; di navbera 8.41 û 16,80yan de, nirxê testa t yê aîdî rehenda duyem di navbera 5.44 û 13,78an de û nirxê testa t yê aîdî rehenda sêyem di navbera 7.44 û 12,98an de hate dîtin. Bi qasî ku (Jöreskog û Sörbom, 2000; Kline, 2011) pêş dixin, nirxê t eger di asta .01ê de nirxeke ji 2.58an mezintir standibe watedar e, di asta .05ê de nirxeke ji 1.96an mezintir standibe watedar e. Nirxê t yê ku ji encama AFRyê de bi dest ketiye ji bo hemû maddeyên sê rehend yên ji encama AFKyê bi dest ketine di asta .01ê de watedar in. Eger nirxê t yê bi dest ketî watedar nebe divê madde ji analîzê bê derxistin (Byrne, 2010). Dema nirxê t hate nirxandin, diyar bû ku piştî encama AFRyê maddeyeke ji modele bê derxistin tuneye. 
Tablo 5: Ji bo PHZSyê Nirxên 't-test'ê yên ji AFRyê Bi Dest Ketine

\begin{tabular}{|l|l|l|l|l|l|l|l|}
\hline Jimara Mad. & t & Jimara Mad. & t & Jimara Mad. & t & Jimara Mad. & t \\
\hline M1 & $12.51^{*}$ & M13 & $12.97^{*}$ & M21 & $9.60^{*}$ & M29 & $15.46^{*}$ \\
\hline M2 & $13.78^{*}$ & M14 & $10.17^{*}$ & M22 & $12.30^{*}$ & M30 & $12.22^{*}$ \\
\hline M3 & $10.07^{*}$ & M15 & $5.44^{*}$ & M23 & $9.94^{*}$ & M31 & $12.98^{*}$ \\
\hline M5 & $10.24^{*}$ & M16 & $8.24^{*}$ & M24 & $7.44^{*}$ & M32 & $14.23^{*}$ \\
\hline M6 & $9.53^{*}$ & M17 & $7.19^{*}$ & M25 & $11.85^{*}$ & M33 & $12.15^{*}$ \\
\hline M7 & $8.93^{*}$ & M18 & $7.48^{*}$ & M26 & $10.77^{*}$ & M34 & $16.80^{*}$ \\
\hline M8 & $12.59^{*}$ & M19 & $12.42^{*}$ & M27 & $12.48^{*}$ & M35 & $13.81^{*}$ \\
\hline M11 & $10.60^{*}$ & M20 & $0.85^{*}$ & M28 & $8.41^{*}$ & & \\
\hline *p $<01$ & & & & & & & \\
\hline
\end{tabular}

Di encama AFRyê de em dibînin ku, binyata sê rehendî ya ji encama AFKyê bi dest ketiye hatiye piştrastkirin. Bi gotineke din; tê dîtin ku, binyata me ya ku xwe dispêre têgerîna lîteraturê û wisa pêk hatiye, piştrast bûye. Modela ku di encama AFRyê de pêk hatiye di Şiklê 2em de hatiye pêşkeşkirin.

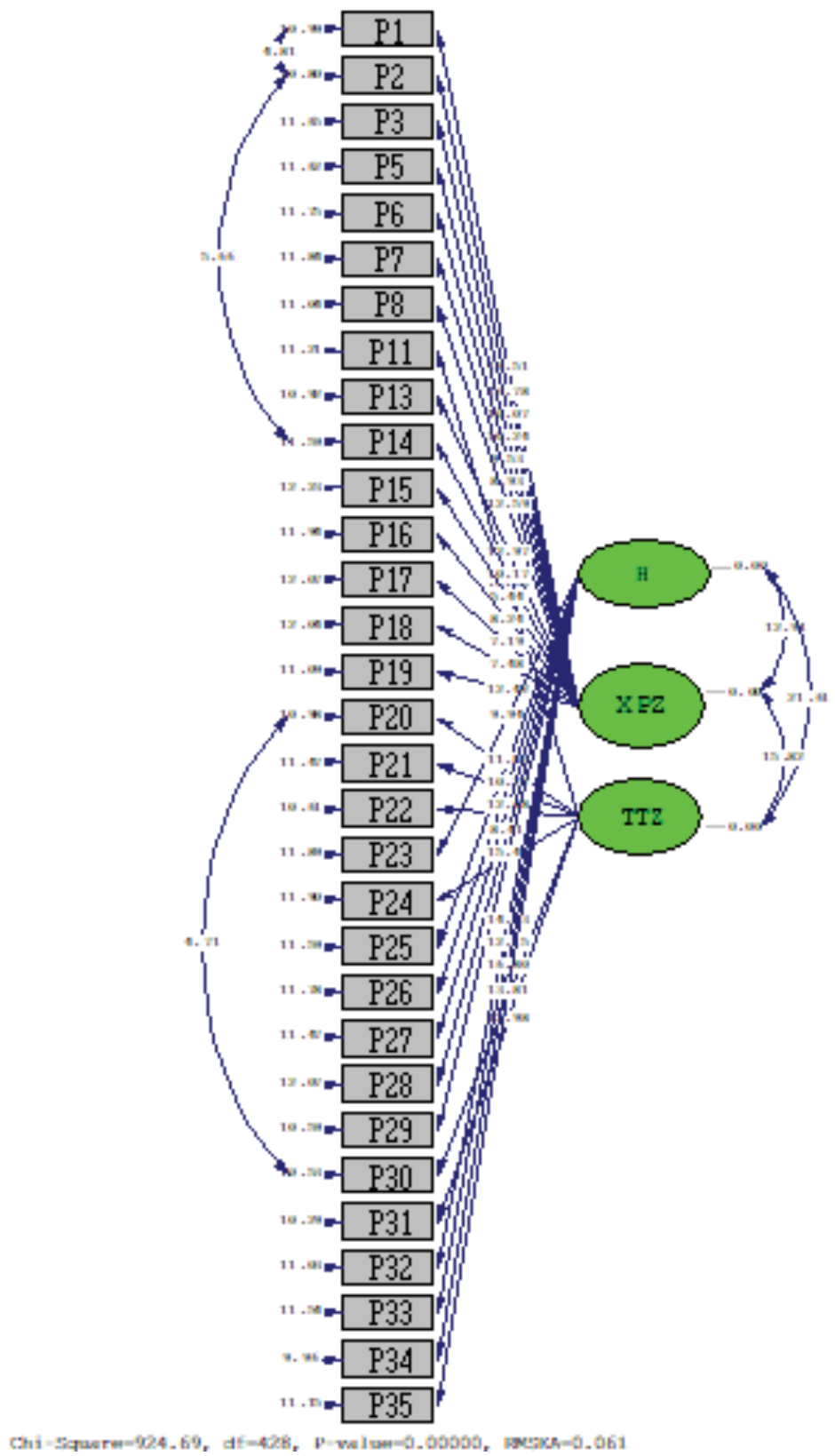

Şeklê 2em: Modela Pîvanê ya Têkilî PHZSyê 


\subsubsection{PÊBAWERÎ}

Qatjimara alfa ya bin rehendên PHZSyê bi rêzê, 0.872, 0.885 û 0.802 ne û qatjimara alfayê ji bo hemû maddeyên pîvanekê wekî 0.915 hatiye hesabkirin. Dema li qatjimarên alfayê yên pîvanekê bi dest ketine bê nêrîn, ew ê bê dîtin ku qatjimara pêbaweriyê berz derketiye. Li gorî van vedîtinan dikare bê gotin ku pîvanek pêbawer e.

\subsubsection{ANALÎZA MADDEYAN}

Ji bo ku hêza texmînê ya pûanê sercem bê diyarkirin û jevkariya maddeyî bê çespandin, korelasyona sercem ya maddeye ya serastkirî hatiye hesabkirin. Herwiha grûpên ser-binî yên \%27î hatine rûberûkirin. Piştî encama analîza maddeyan tesbîtên ku derketine di Tabloya 6an de hatine dayîn.

Tablo 6: Encamên Analîza Maddeyan yên PHZSyê

\begin{tabular}{|c|c|c|c|c|c|c|c|}
\hline 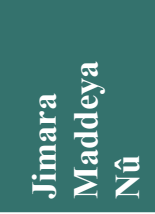 & 章 & 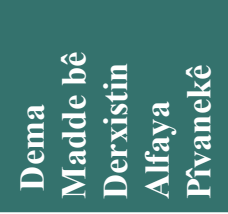 & 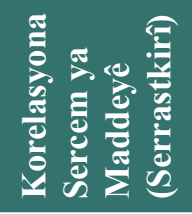 & $\sum_{\frac{2}{2}}^{e}$ & 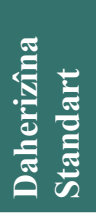 & 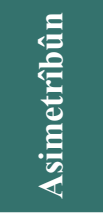 & - \\
\hline M23 & M19 & .912 & .485 & 3,92 & 1.36 & -.96 & $-9.67 *$ \\
\hline M25 & M21 & .912 & .519 & 4.01 & 1.10 & -.93 & $-9.97 *$ \\
\hline M26 & M22 & .912 & .462 & 4.10 & 1.19 & -1.09 & $-10.65 *$ \\
\hline M27 & M23 & .912 & .476 & 4.48 & .94 & -2.04 & $-8.17 *$ \\
\hline M28 & M24 & .914 & .361 & 4.11 & 1.22 & -1.24 & $-6.72 * d s=84$ \\
\hline M29 & $\mathrm{M} 25$ & .911 & .622 & 4.25 & .96 & -1.27 & $-11.87 * * p<.01$ \\
\hline M32 & M28 & .910 & .613 & 4.09 & 1.13 & -1.00 & $-16.50^{*}$ \\
\hline M33 & M29 & .913 & .459 & 4.48 & .92 & -1.94 & $-7.41^{*}$ \\
\hline M34 & M30 & .910 & .655 & 4.26 & 1.01 & -1.14 & $-12.53 *$ \\
\hline M35 & M31 & .912 & .541 & 4.42 & .97 & -1.91 & $-8.91 *$ \\
\hline M1 & M1 & .911 & .553 & 4.58 & 1.13 & -.43 & $-13.24 *$ \\
\hline M2 & M2 & .910 & .599 & 3.97 & 1.26 & -.89 & $-13.70 *$ \\
\hline M3 & M3 & .912 & .459 & 3.80 & 1.29 & -.51 & $-10.74 *$ \\
\hline M5 & M4 & .913 & .435 & 4.27 & 1.07 & -1.27 & $-7.74 * d s=84$ \\
\hline M6 & M5 & .913 & .453 & 4.48 & .88 & -1.95 & $-7.64 * * \mathrm{p}<.01$ \\
\hline M7 & M6 & .913 & .441 & 3.89 & 1.40 & -.92 & $-11.48^{*}$ \\
\hline M8 & M7 & .910 & .597 & 3.55 & 1.42 & -.30 & $-15.89 *$ \\
\hline M13 & M9 & .910 & .611 & 4.11 & 1.20 & -1.20 & $-12.69 *$ \\
\hline M14 & M10 & .912 & .455 & 4.19 & 1.21 & -1.21 & $-9.85 *$ \\
\hline M15 & M11 & .917 & .258 & 3.23 & 1.50 & -.17 & $-5.56^{*}$ \\
\hline M16 & M12 & .913 & .426 & 4.04 & 1.30 & -1.15 & $-9.55^{*}$ \\
\hline M17 & M13 & .915 & .341 & 3.92 & 1.40 & -.94 & $-7.47 *$ \\
\hline M18 & M14 & .912 & .413 & 4.13 & 1.20 & -1.10 & $-9.18 *$ \\
\hline M19 & M15 & .910 & .622 & 4.04 & 1.25 & -.99 & $-13.58 *$ \\
\hline M11 & M8 & .911 & .524 & 3.87 & 1.25 & -.76 & $-13.23 *$ \\
\hline M20 & M16 & .911 & .574 & 3.42 & 1.47 & -.25 & $-15.40 * \mathrm{ds}=84$ \\
\hline M21 & M17 & .912 & .466 & 3.79 & 1.27 & -.74 & $-8.92 * * \mathrm{p}<.01$ \\
\hline M22 & M18 & .911 & .549 & 3.83 & 1.22 & -.70 & $-11.67^{*}$ \\
\hline M24 & M20 & .912 & .336 & 3.41 & 1.38 & -.29 & $-7.52 *$ \\
\hline M30 & M26 & .911 & .552 & 3.80 & 1.22 & -.48 & $-13.61^{*}$ \\
\hline M31 & M27 & .911 & .570 & 3.89 & 1.14 & -.62 & $-14.56^{*}$ \\
\hline
\end{tabular}


Dema ku em li daneyên Tabloya 6an dinerin, em dibinin ku; nirxê t yê aîdî ferqa navbera pûanên maddeyê yê aîdî beşdarên \%27 yên grûpên ser û binî, di rehenda yekem de, di navbera 8,41 û 16,80an de $(\mathrm{ds}=84, \mathrm{p}<0.01)$, di rehenda duyem de, di navbera 5,44 û 13,78an de $(\mathrm{ds}=84, \mathrm{p}<0.01)$ û di rehenda sêyem de, di navbera 7,19 û 12,42yan de $(\mathrm{ds}=84, \mathrm{p}<0.01)$ dugiherin. Di Tabloya 6an de, di navbera korelasyona sercem ya maddeyê de, di faktera yekem de, di navbera .361 û .655an de, di faktera duyem de, di navbera .258 û .622yan de û di faktera sêyem de, di navbera .336 û .574an de rêz dibe. Dema madde têne ravekirin, tê îfadekirin ku nirxên qatjimara korelasyona sercem ya maddeyê eger .30 û li ser be bes e ji bo taybetiyên têne pîvandin ji hev bike (Büyüköztürk, 2010; Erkuş, 2012). Em gihiştine wê encamê ku hemû maddeyên pîvanekê ew ê vê taybetiyê li xwe bigirin. Di raberkirina grûpa \%27 ya ser-bin de, watedarbûna nirxê t nişan dide ku madde ji hev ker e (Erkuş, 2012). Li gorî encamên ji analîza maddeyan bi dest ketine, em dikarin bêjin hemû maddeyên pîvanekê ji hev ker in.

\section{RABERî Û ENCAM}

Di vê lêkolînê de me armanc kir ku amûreke pîvandinê pêş bixin ji bo ku bi awayekî têbar û pêbawer em bikarin helwesta beşdarên duziman bipîvin ya têkilî zimanê wan yê pêwendiyê ku zimanê serî yê wan beşdaran ne tirkî ye. Bona vê, me pîvanekeke wekî Pîvaneka Helwesta Ziman ya Kesane (ji bo duzimanan) pêş xist. Ji bo vê pêşî me hewzeke maddeyan çêkir. Paşê, di derbarê têbariya xuyanî û têbariya hewanî ya pîvanekê de û derbarê zimanê maddeyan de me fikir û nêrînên sê pisporên qadê wergirtin. Piştî nêrîn û pêşniyarê pisporan me pîvanekeke 35 maddeyî saz kir. Maddeyên pîvanekê bi şiklê pileyî ne û pênc vebjêrkên ji tîpa Likert pêk tên wekî; "Qet Ne Wisa ye" (1), "Ne Wisa ye" (2), "Ne Erê Ne Na" (3), "Wisa ye" (4), "Bi Temamî Wisa ye" (5).

Piştî ku pîvanek amade bû, daneyên ku jê hate girtin bi sepandina AFK û AFRyê ve têbariya wan hate testkirin. Di encama AFKyê de têderket ku pîvanek \%45.569ê varyansa sercem îzah dike, ji 31 maddeyan pêk tê û di binyatê de maddeyên xwe di sê fakteran de kom dike. Dema em bala xwe didine ser naverok û binyata teorîk ya maddeyan, di encama analîza fakterî de rehendên bi dest ketine bûne sê grûp. Ji van faktera yekemîn wekî Hestyarî (H), faktera duyemîn wekî Xem û Prestîja Ziman (XPZ) û faktera sêyemîn jî wekî Tercîh û Têrayiya Ziman (TTZ) hate binavkirin.

Li dû diyarbûna rehendên pîvanekê bi encama AFKyê, AFR hate kirin ji bo em bizanin ka modela ji encama AFKyê bi dest ketiye ew ê bê piştrastkirin yan na. Piştî analîza AFRyê derket ku îndeksên lêhatinê yên sê rehendî yên aîdî PHZSyê guncaw in. Di analîza fakterî de \%32 û li ser wekî pîveka nirxê varyansê hate girtin. Di encama AFRyê de, maddeyên ku nirxê varyansa wan .32 ne îndeksên wan yê lêhatinê guncaw hate dîtin. Di encama AFK û AFRyê de hate fêmkirin ku PHZSyê çespandiye ku têbariya wê ya binyatî heye.

Encamên ku ji PHZSyê hatine wergirtin, bi rêbaza pêbaweriya nav-hevgiriyê (qatjimara Cronbach Alfa ya pêbaweriyê) hate testkirin û korelasyona sercem ya maddeyê hate vekolan. Ji bo tebariya pîvekî ya daneyên pîvanekê ferqa di navbera pûanên grûpên ser-bin yên \%27î bi bikaranîna 't-test'a nimûneya serbixwe hate analîzkirin.

Pêbaweriya Cronbach Alfa ya pîvanan, di rehenda Hestyarî (H)yê de 0.872, ji bo rehenda Xem û Prestîja Ziman (XPZ) 0.885 û ji bo rehenda Tercîh û Têrayiya Ziman(TTZ) wekî 0.802 hate hesabkirin. Ji bo ku pîvandinên hatine kirin wekî pêbawer bê dîtin, tê parastin ku qatjimara pêbawariyê .70 û li ser be (Fornell û Larcker, 1981; Tezbaşaran, 1997; Nunnaly û Bernstein, 1994).

Digel vê, ji bo ku hêza texmîna sercema pûanan ya maddeyên aîdî PHZSyê bê diyarkirin û asta jihevkeriya maddeyê bê çespandin analîza maddeyê hate kirin. Dema analîza maddeyê hate kirin, grûpên ser-binî yên \%27î hatine rûberûkirin û korelasyona sercem ya maddeyê ya serastkirî hate vekolan.

Di encama analîza maddeyan de, encamên korelasyona sercem ya maddeyan ya serastkirî di bin rehenda Hestyarî (H)yê de, di navbera .361 û .655an de; di bin rehenda Xem û Prestîja Ziman (XPZ) di navbera .258 û .622yan de; di bin rehenda Tercîh û Têrayiya Ziman (TTZ) de jî di navbera .336 û .574an de nirx wergir- 
tine. Herwiha hate der ku nirxê t yê têkilî ferqên navbera grûpên ser-binî yên \%27î ji bo hemû maddeyên pîvanekê watedar e. Di encama tesbît û vedîtinên bidestketî de derket ku PHZS têbar û pêbawer e.

Divê em vê jî bejin ku di encama têgerîna lîteraturê de di vê biwarê de em rastî tu lêkolînekê nehatin. Em wisa dipên ku di lîteraturê de, PHZS, ew ê vê valahiyê dagire.

\section{JÊDER}

Baltac1, Ali. "Nitel Araştırmalarda Örnekleme Yöntemleri ve Örnek Hacmi Sorunsalı Üzerine Kavramsal Bir İnceleme”, Bitlis Eren Üniversitesi Sosyal Bilimler Enstitüsü Dergisi, cilt.7, sayı.1, (Haziran 2018), s. 231-274.

Bandura, A. "Self-Efficacy Mechanism in Human Agency", American Psychologist, v. 37, n. 2, (1982), p. 122- 47.

Büyüköztürk, Şener. Sosyal Bilimler İçin Veri Analizi El Kitabı, Ankara: Pegem Akademi Yayınları, 2010.

Byrne, B. M. Structural Equation Modeling with AMOS: Basic Concepts, Applications and Programming, NY: Taylor and Francis Group, 2010.

Cattell, R. B. The Scientific Use of Factor Analysis in Behavioral and Life Sciences, New York: Plenum, , 1978.

Cummins, J. Language, Power and Pedagogy: Bilingual Children in the Crossfire, Clevedon: Multilingual Matters, 2000.

Erkuş, A. Psikolojide Ölçme ve Ölçek Geliştirme, Ankara: Pegem Akademi Yayınları, 2012.

Floyd, F. J. - Widaman, K. F. "Factor Analysis in the Development and Refinement of Clinical Assessment İnstruments", Psychological Assessment, 7(3), (1995) p. 286-199.

Fraenkel - Wallen - Hyun, , How to Design and Evaluate Research in

Education (8. b.), New York: McGraw-Hill, 2012.

Garett, P. Attitudes to Language, UK: Cambridge University Press, 2010.

Gorsuch, R. L. Factor Analysis (2nd ed.), NJ: Lawrence Erlbaum, Hillsdale, 1983.

Henson, R. K. - Roberts, J. K. "Use of Exploratory FactorAnalysis in Published Research: Common Errors and Some Commenton Improved Practice", Educational and Psychological Measurement, 66(3), (2006), p. 393-416.

Hickey, Raymond. Language and Society, UK: Cambridge University Press, 2007.

Kıran, Abdullah. Ferhenga Zanistên Civakî, Stenbol: Weşanên Nûbihar, Çapa Yekem, 2014.

Kline, R. B. Principles and Practice of Structural Equation Modeling, New York: The Guilford Press, 2011.

Köpke, B. - Schmid, M. S. "First Language Attrition”, The Next Phase. In: Schmid, M.S. - Köpke, B. - Keijzer, M. - Weilemar, L. (Ed). First Language Attrition: Interdisciplinary Perspectives On Methodological Issues, Amsterdam/Philadelphia: John Benjamins, 2004.

İlhan, M. - Çetin, B. "Sınıf Değerlendirme Atmosferi Ölçeğinin (SDAÖ) Geliştirilmesi: Geçerlik ve Güvenirlik Çalışması”, Eğitim ve Bilim, 39(176), (2014), s. 31-50.

Jöreskog, K. - Sörbom, D. LISREL [Computer Software], Lincolnwood, IL: Scientific Software, Inc, 2000.

Schermelleh-Engel, K. û yd. "Evaluating the Fit of Structural Equation Models: Test of Significance and Descriptive Goodness-of-fit Measures", Methods of Psychological Research-Online, 8(2), 2003, p. 23-74.

Stevens, J. Applied Multivariate Statistics for the Social Sciences (4th Edition), NJ, Mahwah: Lawrence Erlbaum Associates, 2002.

Tabachnick, B. G. - Fidell, L.S. Using Multivariate Statistics (5th ed.), New York: Allyn and Bacon, , 2007.

Trask, R.L. Key Concepts in Language and Linguistics, (ed. Stockwell, P.), Oxon: Routledge, 2007.

Young, D. J. “Creating a Low-anxiety Classroom Environment: What does the Language Anxiety Research Suggest?” Modern Language Journal, 75, (1991), p. 425-439.

Zeraq, Dilawer - Wenda, Mem. Ferhenga Termên Matematîkê, Amed: Weşanên Lîs, 2015. 


\begin{tabular}{|c|c|c|}
\hline \multicolumn{3}{|c|}{ FERHENG JI BO VÊ XEBATÊ } \\
\hline Kurdî & Îngilizî & Tirkî \\
\hline Analîza fakterî & Factor analysis & Faktör analizi \\
\hline Analîza fakterî ya keşfî & Explatory factor analysis & Açımlayıcı faktör analizi \\
\hline Analîza fakterî ya rasteder & Confirmatory factor analysis & Doğrulayıcı faktör analizi \\
\hline Asîmetrîbûn & Skewness & Çarpıklık \\
\hline Barê fakterî yê maddeyê & Item factor loading & Madde faktör yükü \\
\hline Daherizîna standart & Standart deviation & Standart sapma \\
\hline Hokar/fakter & Factor & Faktör, etken \\
\hline Hevgiriya navîn, nav-hevgirî & Internal validity & İç tutarlılık \\
\hline Îndeksên lêhatinê/guncaniyê & Fit indices & Uyum indeksleri \\
\hline Korelasyona sercem ya maddeyê & Item total correlation & Madde toplam korelasyonu \\
\hline Nîvtev & Mean & Ortalama \\
\hline Pêbawerî & Reliability & Güvenirlik \\
\hline Pêkhên & Component & Bileşen \\
\hline Pirsiyar & Question & Sorun \\
\hline Pîvanek & Scale & Ölçek \\
\hline Pîvek & Criteria & Ölçüt \\
\hline Raber & Towards & Karş1 \\
\hline Raberî & Discussion & Tartışma \\
\hline Rehend & Dimension & Boyut \\
\hline Raste & Line & Doğru \\
\hline Rasteyî & Linear & Doğrusal \\
\hline Têbarî & Validity & Geçerlik \\
\hline Têbariya binyatî & Construct validity & Yapı geçerliliği \\
\hline Têbariya hewanî & Content validity & Kapsam geçerliliği \\
\hline Têbariya xuyayî & Face validity & Görünüş geçerliliği \\
\hline Xêzegrafik & Line graph & Çizgi grafiği \\
\hline Xware & Curve & Eğri \\
\hline
\end{tabular}


Pêvek: Forma Pîvaneka Helwesta Zimanê Serî
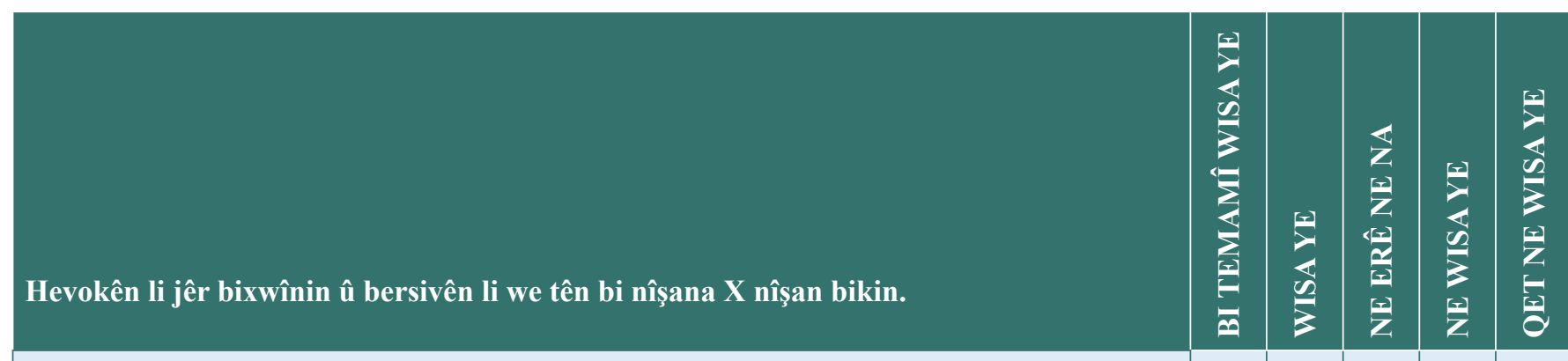

1. Dema ez bi kurdî diaxivim (xeber didim) endîşe dikim ku ez ê xeta bikim.

2. Dema ez bi kurdî diaxivim ditirsim ew ê bi min bikenin.

3. Ew ên zimanê wan yê serî wekî yê min e, ez bi wan re bi zehmetî pêwendiyê datînim.

4. Ez bi kurdî naxivim çunkî eger ez biaxivim însanên din ew ê bi nebaşî berê xwe bidine min.

5. Ez bawer im ku axaftina bi kurdî ji bo min ew ê ne baş be.

6. Li gorî min divê meriv pêşî zimanê fermî yê welatê lê dijî hîn be.

7. Ku kurdî hatibe pirsîn jî ez carna bersivên tirkî didim.

8. Ez bi tirkî difikirim paşê bi kurdî dibêjim.

9. Ez tenê eger mecbûr bimînim bi kurdî diaxivim.

10. Ez ditirsim dema ez kurdî biaxivim hevalên min yên kurdiya wan baş in, ew ê bi min bikenin.

11. Ez ne bawer im axaftina bi kurdî ew ê statuyeke baştir bide min li Tirkiyeyê.

12. Ez bi tirkî diaxivim, malbat û hevalên min kêfxweș dibin.

13. Eger ez bi zimanê xwe yê serî neaxivim, nexwînim û nenivîsim, ew ê nebe kêmasiyeke muhîm di jiyana min de.

14. Eger ez tirkî baş bizanibim, ev bes e ji bo jiyana min ya li Tirkiyeyê.

15. Dema ez bi malbata xwe re me li ciyên fermî (wekî; mekteb, kargeh, civîn, banka, pirtûkxane, mixaze, avahiyên îdarî) ez dixwazim bi wan re tirkî biaxivim.

16. Ez hestên xwe bi tirkî baştir îfade dikim.

17. Kurdiya min nebaş be jî ez rehet im dema li pêş însanan kurdî diaxivim.

18. Li mektebê bi hevalên xwe yên kurd re ez axaftina kurdî tercîh dikim.

19. Li malê ez bi dê û bavê xwe re yan jî ehlê malê re axaftina bi kurdî tercîh dikim ew bi tirkî bikarin biaxivin jî.

20. Ez pêşî bi kurdî difikirim paşê bi tirkî dibêjim.

21. Ez bi kurdî diaxivim, malbat û hevalên min kêfxweş dibin.

22. Ez wisa hîs dikim ku ew ê bibe zirareke mezin eger ez nikaribim bi kurdî biaxivim, bixwînim û binivîsim.

23. Mihefezekirina zimanê min yê dayikê ji bo min muhîm e.

24. Ez bi kurdî baş diaxivîm berî ku ez diçûme mektebê.

25. Çendî xeta û kêmasiyên min hebin jî ez axaftina kurdî tercîh dikim.

26. Ez hestên xwe bi kurdî baştir îfade dikim.

27. Di her firsendê de, kengê dibe bila bibe ez zimanê xwe yê serî diaxivim.

28. Dema ez bi malbata xwe re me li ciyên fermî (wekî; mekteb, kargeh, civîn, banka, pirtûkxane, mixaze, avahiyên îdarî) ez dixwazim bi wan re kurdî biaxivim.

29. Ez ji gelê kurd û çanda kurdan hez dikim.

30. Ez bêhtir rehet im dema ez bi kurdî diaxivim.

31. Ez ji xwendina kurdî hez dikim. 


\section{EXTENDED ABSTRACT}

\section{KURTEYA BERFIREH}

Language attitudes are opinions, ideas and prejudices that speakers have with respect to a language. It is acknowledged that attitude towards a language has an effect on a to learn it faster or inhibit the learning. The main purpose of this study to gain a valid and reliable measurement scale that can be utilized to categorize bilingual individuals' attitude towards their mother tongue. The scale research was applied to a total of 310 Kurdish-Turkish bilingual participants aged between 16-65 living in Van City -Turkey. An exploratory factor analysis and a reliability analysis were performed to determine the validity of the scale..

\section{METHOD}

Personal language attitude scale, native language is Kurdish, was developed to determine the attitude towards language preferences. The item pool formed as a result of the literature research was applied to the participants who have two languages. In order to determine whether the items in this form will measure the language preferences of bilingual participants appropriately and whether they are understood as a language, it was presented to the opinions of three experts. Corrections were made in line with the feedback from field experts and a form consisting of 35 items was finalized. The prepared form consists of "Absolutely Disagree" (1), "Disagree" (2), "Undecided" (3), "Agree" (4), "Completely Agree" (5). Accordingly, the high score indicates that the Personal language attitude is high.

Statistical analysis was performed to reveal the characteristics of the measurements made and the MTAS was applied to the participants. Exploratory factor analysis (EFA) was conducted to examine the construct validity of the developed MTAS and to reveal the factor structure of the scale. Before starting the factor analysis, it should be evaluated whether the data will be suitable for EFA. First, the sample size was checked. There is no definite judgment among researchers regarding the sample size for EFA (İlhan \& Çetin, 2014). However, Cattell (1978) suggests that 200 participants are sufficient, its application to 500 participants is a very good sample, and 3 to 6 times the number of items in the scale is sufficient. In addition, it suggests that with the increase in the number of participants, the items will contribute to the factor structure becoming more pronounced, but 5 times the number of participants is a sufficient sample size for EFA (Stevens, 2002; Gorsuch, 1983). Therefore, the sample size was considered to be suitable for EFA. MTAS was made up of 35 items and applied to 310 participants.

\section{RESULTS}

In this section, information about the validity and reliability of the "Mother Tongue Attitude Scale for Bilingual Individuals (MTAS)" is given. EFA 
and CFA were conducted to determine the construct validity of the Personal Language Attitude (for bilinguals) Scale (PLAS).

\section{EXPLORATORY FACTOR ANALYSIS (EFA)}

Exploratory factor analysis was performed to determine the construct validity of the PLAS and to determine item factor loadings. Exploratory factor analysis was calculated using the Kaiser-Meyer-Olkin (KMO) coefficient and Bartlett Sphericity Test to determine the appropriateness. The KMO value was found to be .91 and the result of the Bartlett test $\left(\chi^{2}=3949,540, p=\right.$ 0.000 ), which tested the multivariate normality, was found to be significant. It was concluded that the resulting factor analysis was appropriate.

As a result of the factor analysis, it was seen that the items with eigenvalues greater than 1 were collected in 6 factors. It was observed that the items collected in these 6 factors explained about $56,521 \%$ of the scale. In the exploratory factor analysis, he emphasized that the factor loads of the items should be above 0.30 (Floyd \& Widaman, 1995; Tabachnick \& Fidell, 2007). In the factor analysis, the item load value was below 0.30 and the items that could not be loaded were excluded from the analysis. As a result of the factor analysis, it was decided to collect three factors for the items. The properties of the items related to the repeated EFA results are presented in Table 1.

\section{CONCLUSION AND DISCUSSION}

In this study, it was aimed to develop a measurement tool to measure the attitudes of participants whose native language is not Turkish towards Personal Language attitudes (for bilinguals) in a valid and reliable way. An item pool has been created while developing PLAS. Expert opinion was obtained from three experts on the scope, appearance validity and language of the scale. Considering the recommendations of experts, a scale of 35 items was created. Items in the scale; It has been prepared as a five-point Likert-type scale, such as I Fully Agree (5) $\rightarrow$ Strongly Disagree (1).

The validity of the data obtained from MTAS was tested by applying EFA and CFA. As a result of the EFA, it was concluded that the items were gathered under three factors in terms of structure, consisting of 31 items explaining $45.569 \%$ of the total variance. Considering the content and theoretical structures of the items to the dimensions obtained as a result of factor analysis, the first factor was named Hestyarî, the second factor was named as Xem û prestîja ziman, and the third factor was named Tercîh û têrayiya ziman. CFA was applied to determine whether the model obtained as a result of EFA can be verified or not. As a result of the CFA analysis, it has been concluded that the fit indexes of MTAS, which are obtained in three dimensions, are appropriate. In factor analysis, variance value of $32 \%$ and above was taken as the criterion. As a result of the CFA, fit indexes were found to be suitable for items with a variance value of .32 . As a result of EFA and CFA, it is understood that the structure validity of MTAS is provided.

The internal consistency reliability (Cronbach's Alpha reliability coefficient) method of the results of the measurements obtained from the MTAS was tested and the total item correlations were examined. For the criterion 
validity of the data obtained from the scale, the difference between the total scale scores of the upper-subgroups of $27 \%$ was analyzed using the independent sample t test.

The Cronbach Alpha reliability of the measurements was calculated as 0.872 for the Hestyarî $(\mathrm{H})$ dimension, 0.885 for the Xem û prestîja ziman (XPZ) dimension, and 0.802 for the Tercîh û têrayiya ziman (TTZ) dimension. He argues that the reliability coefficient should be .70 and above for the measurements to be considered reliable (Fornell \& Larcker, 1981; Tezbaşaran, 1997; Nunnaly \& Bernstein, 1994).

Item analysis was carried out in order to determine the predictive power of the items belonging to the MTAS to the total score and to determine the item discrimination levels. While analyzing the item, 27\% lower and upper groups were compared and the corrected total item correlation was examined.

As a result of the item analysis, the corrected item total correlation results were between .361 and .655 in the Hestyarî $(\mathrm{H})$ sub-dimension, between .258 and .622 in the Xem û prestîja ziman (XPZ) sub-dimension, and in the Tercîh û têrayiya ziman (TTZ) sub-dimension. It is seen that it takes a value between 336 and .574 . In addition, the $t$ value related to the differences between the $27 \%$ lower and upper groups was found to be significant for all items in the scale. As a result of the findings obtained, it was concluded that MTAS developed was valid and reliable. No research on this subject has been encountered in the literature research conducted. It is thought that the MTAS will fill this gap in the literature. 